\title{
Manejo conservador de fraturas do complexo zigomático-orbitário: Relato de caso clínico
}

Conservative management of fractures in the zygomatic-orbitary complex: Clinical case report

Manejo conservador de las fracturas del complejo cigomático-orbitario: Reporte de un caso clínico

Recebido: 15/07/2021 | Revisado: 22/07/2021 | Aceito: 24/07/2021 | Publicado: 01/08/2021

Chiara Cristina Diógenes

ORCID: https://orcid.org/0000-0001-6180-9549 Universidade Estadual da Paraíba, Brasil E-mail: chiaracristinadgns@gmail.com

Maxsuel Bezerra da Silva

ORCID: https://orcid.org/0000-0003-4366-9508

Universidade de Pernambuco, Brasil

E-mail: maxsuelmd@ hotmail.com

Ingrid Torres de Almeida

ORCID: https://orcid.org/0000-0002-4661-1849

Universidade de Pernambuco, Brasil

E-mail: ingridtalmeida@gmail.com

Lucas Emmanuell de Morais Neves

ORCID: https://orcid.org/0000-0001-7257-3148

Universidade de Pernambuco, Brasil

E-mail: lucas_emmanuell@hotmail.com

Frank Gigianne Teixeira e Silva

ORCID: https://orcid.org/0000-0001-5115-8586

Faculdade Santa Maria, Brasil

E-mail: frankodonto@gmail.com

Amanda Galvão Souza

ORCID: https://orcid.org/0000-0002-1076-1394

Universidade de Pernambuco, Brasil

E-mail: amandagalvaos@outlook.com

Roberta Karolina Borges de Souza

ORCID: https://orcid.org/0000-0003-4999-1370

Universidade de Pernambuco, Brasil

E-mail: karolina-Borges@hotmail.com

Jessica da Silva Cunha

ORCID: https://orcid.org/0000-0003-1570-2964

Universidade de Pernambuco, Brasil

E-mail: jc.odonto.2018@gmail.com

Sinval Vinícius Barbosa do Nascimento

ORCID: https://orcid.org/0000-0001-6192-6761

Universidade de Pernambuco, Brasil

E-mail: sinvalvinicius@outlook.com

Carlos Augusto Pereira do Lago

ORCID: https://orcid.org/0000-0001-9457-714X

Hospital da Restauração Governador Paulo Guerra, Brasil

E-mail: carloslago1810@ hotmail.com

\section{Resumo}

O zigoma é um osso piramidal que apresenta um corpo espesso e quatro processos, o temporal, orbital, maxilar e frontal. Estes processos são os pontos de fragilidade deste osso. O tratamento das fraturas do complexo zigomático pode ser realizado de forma aberta ou fechada. Uma técnica conservadora bastante utilizada e eficaz é a utilização do Gancho de Barros, sua maior vantagem é a rapidez e eficiência na redução. Paciente do sexo masculino, leucoderma, 52 anos de idade, compareceu ao Hospital da Restauração em Recife/PE, com histórico de agressão física em face do lado esquerdo, cursando com fratura em ossos da face (complexo zigomático-orbitário no lado esquerdo), arco zigomático no lado esquerdo, maxila e em órbita (Blow-out) no lado esquerdo. $\mathrm{O}$ tratamento estabelecido foi a redução anatômica utilizando o Gancho de Barros. A redução fechada com Gancho de Barros neste caso específico, demonstrou ser um artificio seguro e confiável, com bom prognóstico e sem observação de complicações e sequelas pós-operatórias.

Palavras-chave: Fraturas zigomáticas; Fraturas orbitárias; Tratamento conservador. 


\begin{abstract}
The zygoma is a pyramidal bone that has a thick body and four processes, the temporal, orbital, maxillary and frontal. These processes are the weak points of this bone. The treatment of fractures of the zygomatic complex can be performed in an open or closed way. A conservative technique widely used and effective is the use of Gancho de Barros, its biggest advantage is the speed and efficiency in the reduction. Male patient, leucoderma, 52 years old, attended the Hospital da Restauração in Recife / PE, with a history of physical aggression on the left side, with fractures in the bones of the face (zygomatic-orbital complex in the left side), zygomatic arch on the left side, maxilla and orbit (Blow-out) on the left side. The treatment established was anatomical reduction using the Gancho de Barros. The closed reduction with Gancho de Barros in this specific case, proved to be a safe and reliable device, with a good prognosis and without observation of complications and post-operative sequelae.
\end{abstract}

Keywords: Zygomatic fractures; Orbital fractures; Conservative treatment.

\title{
Resumen
}

El cigoma es un hueso piramidal que tiene un cuerpo grueso y cuatro procesos, el temporal, orbitario, maxilar y frontal. Estos procesos son los puntos débiles de este hueso. El tratamiento de las fracturas del complejo cigomático se puede realizar de forma abierta o cerrada. Una técnica conservadora muy utilizada y eficaz es el uso de Gancho de Barros, su mayor ventaja es la rapidez y eficacia en la reducción. Paciente masculino, leucoderma, 52 años, que acudió al Hospital da Restauração de Recife / PE, con antecedente de agresión física en el lado izquierdo, con fracturas en los huesos de la cara (complejo cigomático-orbitario en el lado izquierdo ), arco cigomático en el lado izquierdo, maxilar y órbita (Blow-out) en el lado izquierdo. El tratamiento establecido fue la reducción anatómica mediante el Gancho de Barros. La reducción cerrada con Gancho de Barros en este caso concreto, resultó ser un dispositivo seguro y confiable, con buen pronóstico y sin observación de complicaciones y secuelas postoperatorias.

Palabras clave: Fracturas cigomáticas; Fracturas orbitarias; Tratamiento conservador.

\section{Introdução}

O zigoma é um osso piramidal que apresenta um corpo espesso e quatro processos, o temporal, orbital, maxilar e frontal. Estes processos são os pontos de fragilidade deste osso, em que comumente ocorre a separação pelas suturas que une os ossos unidos a ele. O zigoma é um osso que frequentemente sofre com traumas faciais devido a sua projeção projetada na face, sendo a fratura mais recorrente após as dos ossos nasais (Barros \& Manganello, 2000; Brucoli et al., 2019).

O zigoma tem a ação de dissipar e transmitir forças mastigatórias, sobretudo por meio do pilar zigomático, oferecendo também proteção ao globo ocular (Paulesini Junior, Farias, Aquati, Rapoporat \& Leporace, 2008).

$\mathrm{Na}$ maioria das vezes, as classificações das fraturas faciais oferecem um auxílio para o manejo. Mas as fraturas do complexo zigomático não oferecem contribuições precisas para determinar o tipo de tratamento apropriado. Portanto, a decisão de abordar estas fraturas por meio de redução aberta ou fechada ainda gera diversas controvérsias (Manganello, Silva, Pacheco, 2003; Padmanavam \& Mishra, 2018).

Os principais sinais e sintomas associados a estas fraturas são dormência na área inervada pelo nervo infraorbital, epistaxe, assimetria facial devido ao afundamento da região zigomática, equimose subconjuntival, edema e hematoma palpebral, presença de degrau na região infraorbitária, edema e equimose em mucosa jugal, degrau no pilar zigomático e diplopia (Paulesini Junior, Farias, Aquati, Rapoporat \& Leporace, 2008).

Para um adequado diagnóstico e tratamento dessas fraturas, os exames radiográficos são de suma importância. Dentre eles, destaca-se a radiografia extraoral de Waters e de Hirtz, sendo a de Waters responsável pela observação da fratura de zigoma e o possível velamento do seio maxilar, e a de Hirtz observação do grau de desvio ósseo provavelmente exibido pelo arco zigomático e sua relação com o processo coronóide. Apesar disto, a Tomografia Computadorizada continua sendo o padrão-ouro, pois ela permite avaliar a localização precisa das imagens sem sobreposição (Paulesini Junior, Farias, Aquati, Rapoporat \& Leporace, 2008).

O tratamento das fraturas do complexo zigomático pode ser realizado de forma aberta ou fechada. A fixação interna rígida consiste na utilização de miniplacas e parafusos pode ser realizada em pilar zigomático, sutura frontozigomática e/ou 
pilares caninos. E podem ser utilizadas técnicas incruentas/fechadas que são aquelas em que não há acesso direto a fratura por meio de incisão (Ellis III \& Kittidumkerng, 1996).

A restauração do contorno malar facial, conformação do globo ocular, oclusão dentária, assim como a dimensão de movimento mandibular normal é fundamental e objetivo no tratamento de fraturas do complexo zigomático. O complexo zigomático-orbitário representa quatro pilares fixados por suturas nos ossos frontal, esfenoidal, temporal e maxilar. Dessa forma, o alinhamento anatômico apropriado em todas as quatro linhas de sutura é indispensável para impedir que hajam alterações na aparência facial, conformação do globo ocular, dentre outros prejuízos funcionais. Diversos tratamentos cirúrgicos e métodos de abordagem foram e são apresentados para obtenção de um alinhamento anatômico aceitável e bemsucedido. Mas não existe uma concordância quanto ao número de pontos de fixação, sequências de fixação rígida ou abordagens não cirúrgicas. (Birgfeld, Mundinger, Gruss, 2017; Cohn et al., 2020)

Determinadas fraturas podem se beneficiar da redução e tratamento de forma conservadora/fechada, já as com maior comunicação e deslocamento necessitam de tratamentos mais invasivos para obtenção de um resultado mais aceitável (Jardim, Faverani, Ferreira, Nogueira \& Garcia Júnior, 2011).

As fraturas zigomáticas que são assintomáticas e que não possuem nenhum ou mínimo deslocamento são comumente tratadas com observação. No entanto, as fraturas com deslocamento ou deficiências funcionais ou estéticas, como diplopia, compressão do músculo extraocular, má oclusão e/ou trismo, vão necessitar, na maioria das vezes, de intervenção cirúrgica (Starch-Jensen, Linnebjerg \& Jensen, 2018; Jardim, Faverani \& Ferreira, 2011).

Uma técnica conservadora bastante utilizada e eficaz é a utilização do Gancho de Barros, sua maior vantagem é a rapidez e eficiência na redução. A redução anatômica é feita pela aplicação da força diretamente, contribuindo para maior estabilidade do fragmento após seu reposicionamento anatômico. No entanto, não é recomendada para manejo de fraturas cominutivas ou em que está ocorrendo rotação do osso zigomático para baixo e para dentro (Jardim, Faverani, Ferreira, Nogueira \& Garcia Júnior, 2011).

O objetivo deste estudo é discutir, através de relato de caso, os aspectos atuais do tratamento conservador de fraturas do terço médio e arco zigomático em que este manejo apresentou-se de forma satisfatória.

\section{Metodologia}

O presente ensaio é um estudo intervencional descritivo e qualitativo de relato de caso (Pereira et al.,2018), o qual possui a autorização do paciente por meio do Termo de Consentimento Livre e Esclarecido (TCLE), elaborado em linguagem acessível à compreensão do paciente relatado, evidenciando o destino das informações coletadas do paciente, assim como os benefícios e riscos associados à sua participação que foi assinado no momento de entrada no Hospital. Também foi realizada uma breve revisão da literatura por meio de busca de artigos científicos, revisões sistemáticas, meta-análises e relatos de casos, dos últimos 15 anos, na base de dados PubMed, Scielo e Lilacs, e também consultas a livros bases da Traumatologia.

\section{Relato de Caso}

Paciente do sexo masculino, leucoderma, 52 anos de idade, compareceu ao Hospital da Restauração em Recife/PE, com histórico de agressão física em face do lado esquerdo, cursando com fratura do complexo zigomático-orbitário no lado esquerdo: arco zigomático, maxila e Blow-out.

Ao exame físico extraoral frontal foi notada perda de projeção malar (demonstrado na figura 1), em vista superior notou-se afundamento em região de corpo e arco zigomático em lado esquerdo. Não havia lacerações, edemas e hematomas. Mas o mesmo apresentava uma leve limitação de abertura bucal. 
Research, Society and Development, v. 10, n. 9, e51210918300, 2021

(CC BY 4.0) | ISSN 2525-3409 | DOI: http://dx.doi.org/10.33448/rsd-v10i9.18300

Figura 1: Vista ínfero-frontal.

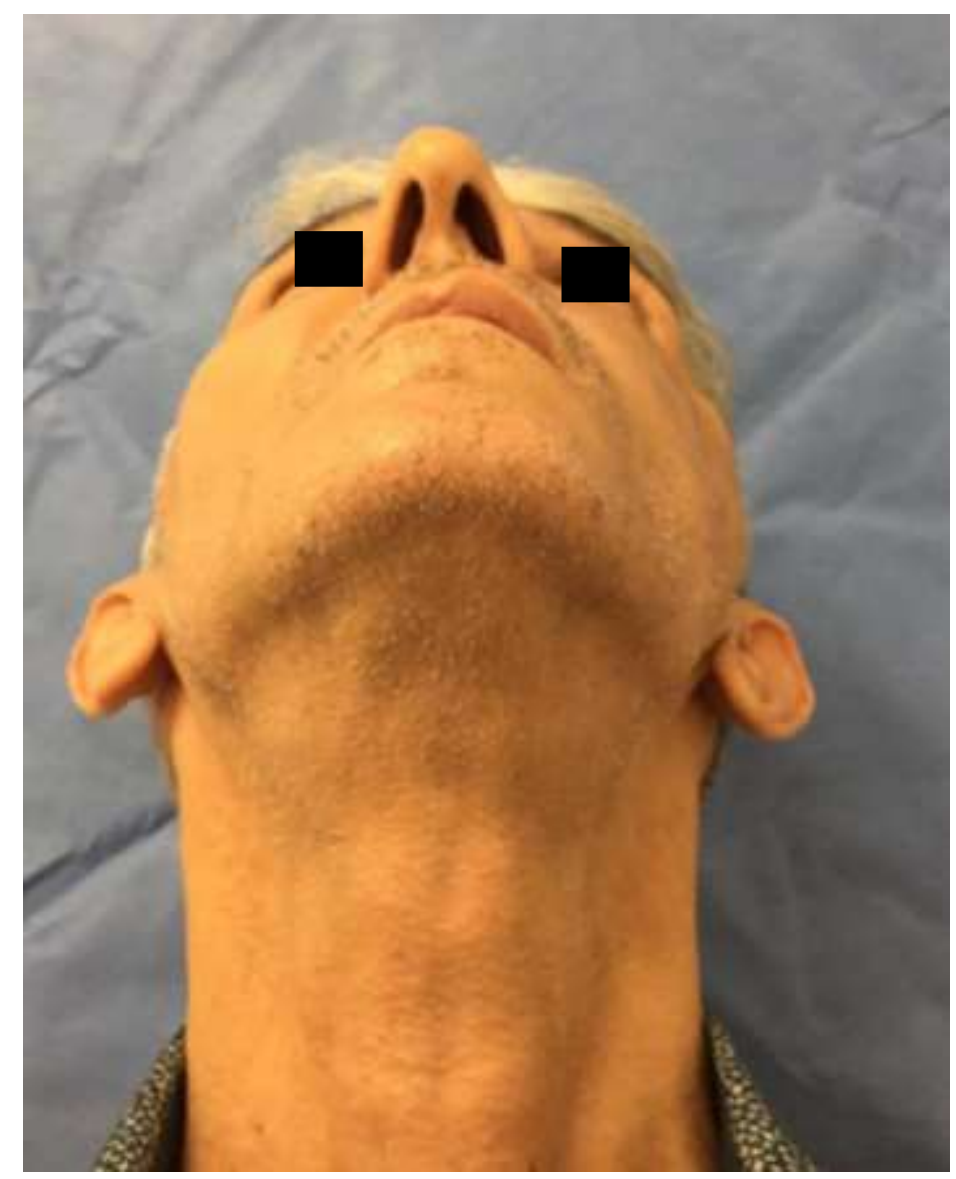

Fonte: Autores.

Ao realizar a Tomografia Computadorizada, nos cortes axiais e coronais foi confirmada fratura do complexo zigomático-orbitário, arco zigomático, maxila e assoalho de órbita transcorrendo com uma fratura blow-out, demonstrados na Figura 2. 
Figura 2: Tomografia Computadorizada em corte axial e coronal pré-operatória.

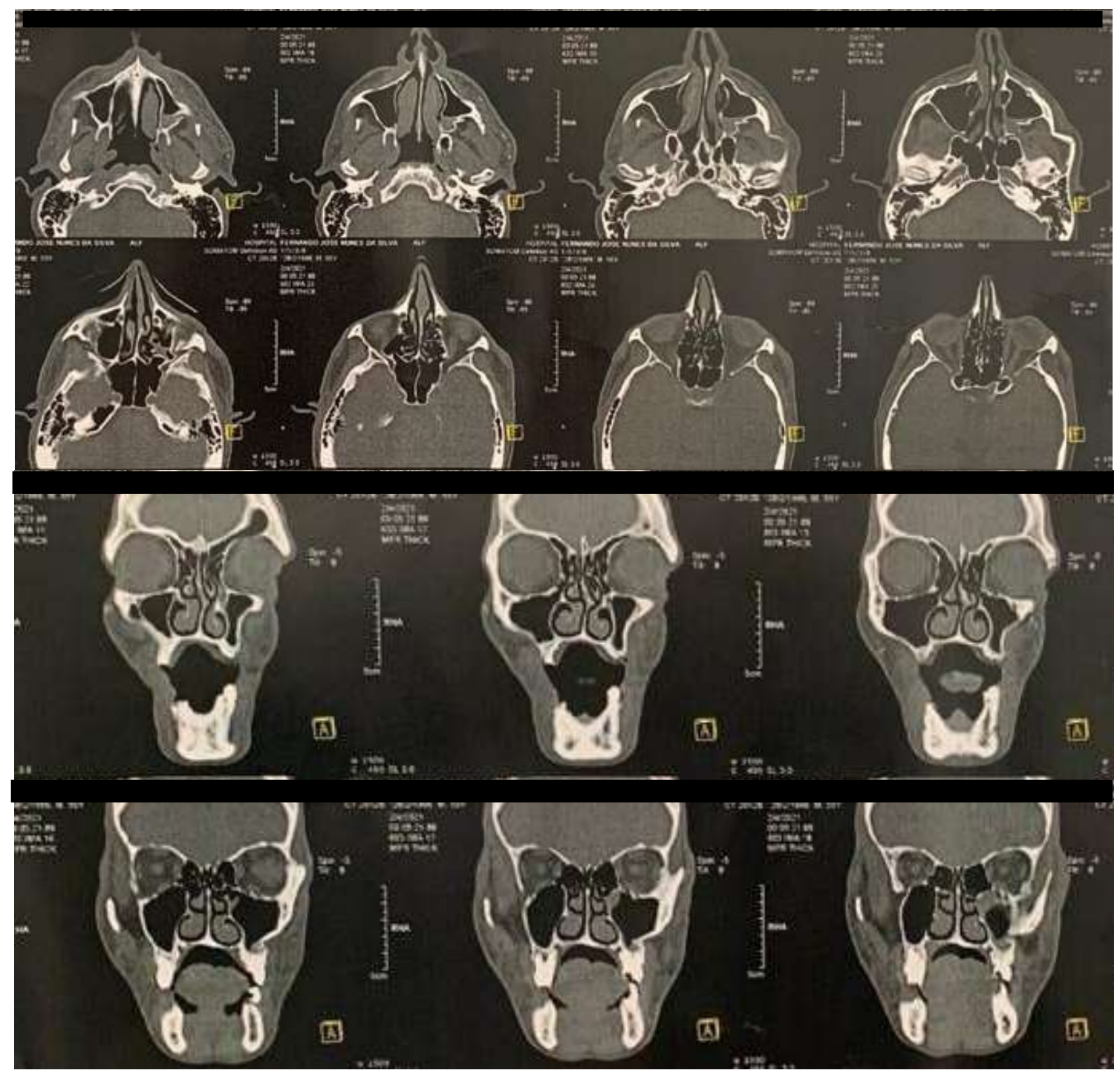

Fonte: Autores.

Devido ao mínimo deslocamento e sem sinais de cominuição, o tratamento proposto foi conservador/incruento, por meio de redução com Gancho de Barros via percutânea. Primeiramente foi realizada antissepsia pré-operatória com clorexidina $2 \%$, anestesia local superficial em alguns pontos específicos da pele, pequena incisão cutânea com bisturi e lâmina 15 no lado acometido apenas para proporcionar a inserção do instrumento. Após isso, realizou-se a inserção do Gancho de Barros à nível da depressão e realizando movimentos de tracionamento para adequada redução. A sutura foi apenas um ponto simples.

A fratura do tipo Blow-out, neste caso, não justificava nenhuma abordagem cirúrgica, pois nas observações clínicas e radiográficas foi notado mínimo deslocamento, e nenhum sinal e sintoma de encarceramento muscular, oftalmoplegia, diminuição da acuidade visual, diplopia, distopia nem enoftalmia.

Em pós-operatório imediato já se pode notar melhora significativa da projeção malar e alinhamento dos cotos ósseos observados na TC pós redução. O paciente foi aconselhado a repousar por um período de 10 dias e evitar novos traumas na região. 
Figura 3: Fotografias pós redução das fraturas.

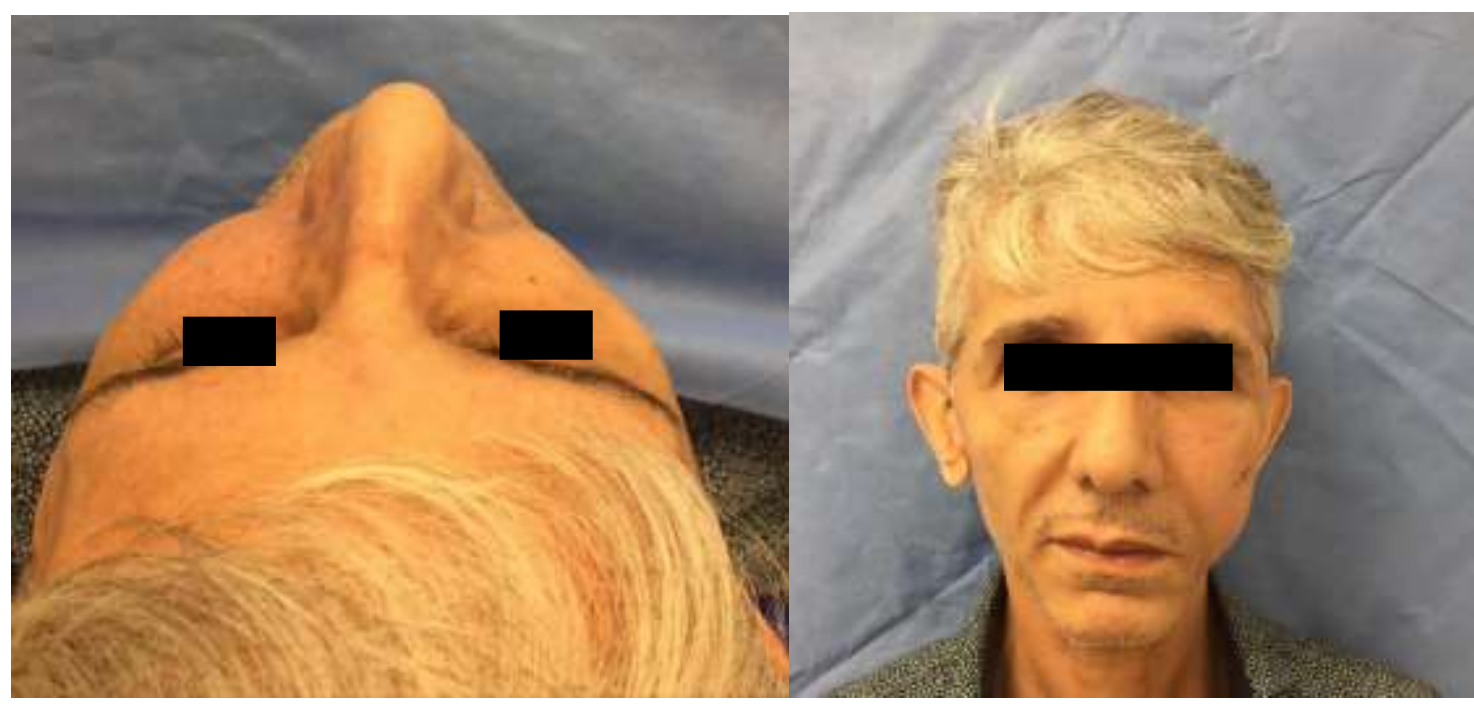

Fonte: Autores.

Figura 4: Tomografia Computadorizada pós-operatória.

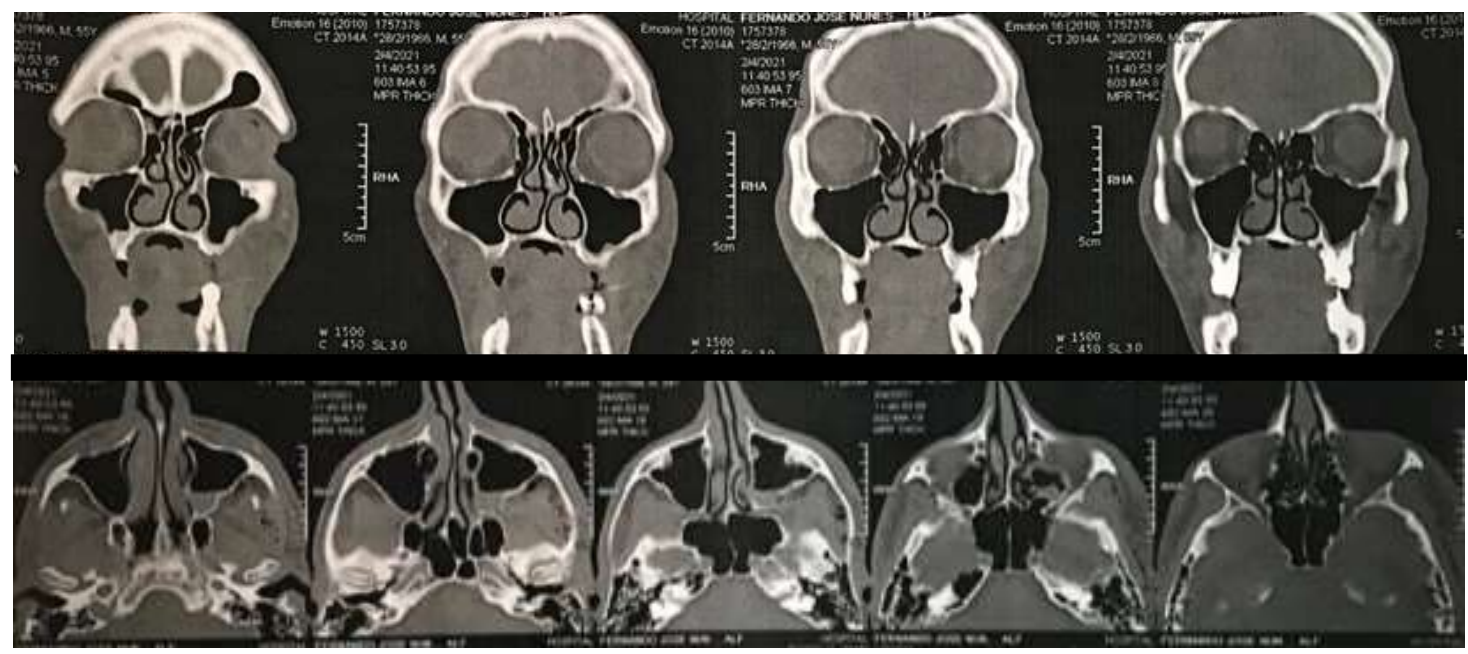

Fonte: Autores.

\section{Discussão}

O complexo zigomático é formado pelo corpo do osso zigomático, arco zigomático e um prolongamento do osso temporal. É um osso piramidal que apresenta quatro processos: temporal, orbital, maxilar e frontal. E estes processos são as zonas de fragilidade deste osso, onde na maioria das vezes ocorre separação na região de suas suturas com os ossos associados. Devido a sua posição proeminente, o zigomático possui funções para tanto para proteção de estruturas, quanto para dar o contorno facial (Manganello, Silva \& Pacheco, 2003; Fonseca, 2000).

Os sinais e sintomas que podem ser encontrados após fratura desse complexo são: limitação de abertura bucal podendo estar associada ao bloqueio do processo coronóide da mandíbula pelos fragmentos do arco zigomático, parestesia na região que o nervo infraorbitário inerva, hematoma, equimose periorbitária, distopia, enfisema, epistaxe, assimetria, hemorragia, enoftalmia, hiposfagma, telecanto traumático, anisocoria, exoftalmia e crepitação óssea (Manganello, Silva \& Pacheco, 2003; Fonseca, 2000). Neste caso, os únicos sinais e sintomas presentes eram a abertura bucal limitada e afundamento da região lateral esquerda do terço médio. E não havia nenhuma complicação orbitária, apesar da presença da fratura Blow-out. 
Em relação a sua epidemiologia, constituem as segundas fraturas mais frequentes dos ossos da face, quando se leva em consideração o corpo do zigoma. No entanto, se considerar apenas o arco zigomático isolado, este é o quarto tipo de fratura mais comum. Dentre as causas mais comuns dessas ocorrências, destaca-se os acidentes de trânsito, agressões físicas e quedas (Brasileiro \& Passeri, 2006). O estudo apresentado demonstra relação com a epidemiologia destas fraturas em prevalência da localização e da etiologia.

Quanto ao sexo de acometimento, há uma maior prevalência do sexo masculino sendo responsável por aproximadamente $80 \%$ dos casos. E a faixa etária mais acometida é dos 21 aos 30 anos (Peron, Ferreira, Camarini, Iwaki Filho, Farah \& Pavan, 2009). Há corroboração deste caso com o sexo masculino, mas não com a idade, pois o paciente apresentava uma idade superior.

As fraturas de arco zigomático ocasionalmente causam depressão malar, em que ocorre uma assimetria estética, enoftalmia, distopia, trismo. A presença da limitação de abertura bucal, é relacionada a proximidade entre o processo coronóide e o arco zigomático. Caso este tipo de fratura não seja tratada adequadamente, podem ocorrer complicações funcionais severas, incluindo abertura bucal prejudicada, anquilose da articulação temporomandibular e paralisia do nervo facial (Hindin, Muetterties, Mehta, Boukovalas, Lee \& Bradley, 2017).

O tratamento cirúrgico atualmente ainda é o escolhido para a maioria dos cirurgiões, tendo uma média de aproximadamente $56 \%$ com acesso cirúrgico e fixação interna rígida. O cirúrgico possui indicação prioritariamente quando existem alterações estéticas e/ou funcionais, tendo a fixação interna rígida em uma ou mais localizações. No entanto, a indicação deste procedimento varia de acordo com fatores como: o tipo de trauma, se as fraturas são simples ou complexas e também a experiência do cirurgião (Peron, Ferreira, Camarini, Iwaki Filho, Farah \& Pavan, 2009).

O tratamento destas fraturas objetiva restituir a anatomia normal dos pilares afetados e melhorar a projeção dos tecidos moles associados e o formato simétrico da órbita. Além do mais, um importante local a ser reduzido é a sutura esfenozigomática, principalmente em fraturas de alta energia, pois é um local que muitas vezes indica se as demais reduções estão em posição correta. O tratamento conservador de redução usando gancho transcutâneo é habitualmente utilizado para as fraturas do complexo zigomático-orbitário que possuem deslocamento mínimo (Giran et al., 2019).

Neste caso em questão, a redução com o gancho de Barros possibilitou a redução do arco zigomático, da região esfenozigomática e da projeção do zigoma que foi melhorada.

O mínimo acesso por meio de uma abordagem que pode ser por acessos extraoral, intraoral ou percutâneo é utilizada para boa parte dos casos de fraturas somente de arco zigomático. No entanto, nestas técnicas, se consegue redução das fraturas por meio de força de tração, podendo levar a consequências como dificuldade de manipulação, especialmente em fraturas que ocorre depressão grave ou tardia, assim como redução inapropriada e fraturas em mais pontos podendo estas últimas ser consequência do excesso e uso descontrolado de força (Litschel \& Suárez, 2015; Cinal et al., 2019). Para isso, é necessário que a abordagem seja de maneira cuidadosa, com força moderada e ordenada, para obtenção de resultados satisfatórios como neste caso clínico.

Portanto, a redução fechada estará melhor indicada em lesões de baixa velocidade, minimamente deslocadas ou não deslocadas e que permanecem estáveis após as tentativas iniciais de redução. O arco zigomático é o mais comumente tratado por este tipo de redução (Bergeron \& Raggio, 2021). Foi devido ao mínimo deslocamento dos pilares faciais e mínimas consequências, que o tratamento estabelecido foi o incruento e este obteve êxito.

Estas fraturas devem ser reparadas antes da cicatrização dos tecidos moles e ósseos. O edema facial, na maioria das vezes, disfarça as deformidades estéticas e eleva a dificuldade de exposição. Idealmente, o reparo das fraturas do complexo zigomático deve ocorrer no período de 7 a 10 dias após a lesão inicial. Quanto mais tarde, mais difícil ficará devido à fibrose e cicatrizes subsequentes (Bergeron \& Raggio, 2021). 
A técnica com gancho de Barros tem como vantagens: execução com anestesia local, incisão e cicatriz mínimas não causando problemas estéticos, rapidez e eficiência na redução pois há uma aplicação direta de força para redução, contribuindo para a estabilidade dos fragmentos após sua colocação na posição normal (Santos Júnior, 1996; Lahmiti et al., 2015).

Em relação aos cuidados pós-operatórios, estes vão variar de acordo com o grau de lesão e dos métodos de reparo escolhidos. Mas apesar disto, o paciente é instruído a evitar atividades fatigantes por pelo menos 14 dias para que haja cura completa e diminuta formação de hematomas e inchaço. Cuidados auxiliares incluem dieta leve e evitar exposição solar. É necessário acompanhar o paciente no pós-operatório para observar quaisquer complicações (Bergeron \& Raggio, 2021). Especificamente para este relato, como cuidados pós-operatórios, o paciente foi aconselhado a retornar no outro dia para avaliação e repousar por 10 dias.

\section{Conclusão}

A redução fechada com Gancho de Barros neste caso específico, demonstrou ser um artificio seguro e confiável, com bom prognóstico e sem observação de complicações e sequelas pós-operatórias. Ademais, a redução incruenta deve ser utilizada sempre que possível, pois esta diminui a morbidade de procedimentos e reduz a oneração ao serviço público.

Ademais, sugere-se que para futuros trabalhos, façam uma abordagem sobre outras formas de tratamento conservador, relacionando os aspectos clínicos aos encontrados na literatura.

\section{Referências}

Barros, J. J. \& Manganello, L. C. S. (2000). Fraturas do complexo zigomático. In: Traumatismo bucomaxilofacial. (2a ed.), Roca; $357-72$.

Bergeron, J. M. \& Raggio, B. S. (2021). Zygomatic Arch Fracture. In: StatPearls [Internet]. Treasure Island (FL): StatPearls Publishing.

Birgfeld, C. B., Mundinger, G. S. \& Gruss, J. S (2017). Medicina baseada em evidências: Avaliação e tratamento de fraturas do zigoma. Plast. Reconstr. Surg. 139 (1): 168e-180e

Brasileiro, B. F. \& Passeri, L. A. (2006). Epidemiological analysis of maxillofacial fractures in Brazil: A 5-Year prospective study. Oral Surg Oral Med Oral Pathol Oral Radiol Endod; 102:28-34.

Brucoli et al. (2019). The "European zygomatic fracture" research project: The epidemiological results from a multicenter European collaboration. $J$ Craniomaxillofac Surg;47(4):616-621

Cinal, H., Barin, E. Z., Çakmak, M. A., Kara, M, Yilmaz, K. \& Tan, O. (2019). Novel Surgical Technique for Repair of Zygomatic Fractures: Lever Technique. Plast Surg (Oakv);27(2):135-140.

Cohn, J. E., Othman, S., Bosco, S., Shokri, T., Evarts, M., Papajohn, P. \& Zwillenberg, S. (2020). Management of Isolated Zygomatic Arch Fractures and a Review of External Fixation Techniques. Craniomaxillofac Trauma Reconstr; 13(1):38-44.

Ellis III, E. \& Kittidumkerng, W (1996). Analysis of treatment for isolated zygomatic maxillary complex fractures. J Oral Maxillofac Surg; 54:386-400.

Fonseca, R. J. (2000). Oral and maxillofacial surgery. Saunders.

Giran, G., Paré, A., Croisé, B., Koudougou, C., Mercier, J. M., Laure, B., Corre, P. \& Bertin, H (2019). Radiographic evaluation of percutaneous transfacial wiring versus open internal fixation for surgical treatment of unstable zygomatic bone fractures. PLoS One; 15;14(8):e0220913.

Hindin, D. I., Muetterties, C. E., Mehta, C., Boukovalas, S., Lee, J., \& Bradley, J. P. (2017). Treatment of isolated zygomatic arch fracture: improved outcomes with external splinting. Plastic Reconstr Surg;139(5):1162e-1171e

Jardim, E. C. G., Faverani, L. P., Ferreira, G. R., Nogueira, L. M. \& Garcia Júnior, I. R (2011). Tratamento conservador de fratura de arco zigomático: uma visão conservadora. Salusvita ;30(1):39-46.

Lahmiti. S., Aziz, Z., Lakouichmi, M., Hiroual, A., Bouaichi, A. \& Mansouri Hattab, N. (2015). Réduction des fractures de l'arcade zygomatique par pince fixe-champ [Towel clip reduction of zygomatic arch fracture]. Rev Stomatol Chir Maxillofac Chir Orale;116(2):92-4

Litschel, R. \& Suárez, G. A (2015). Management of Zygomatic Fractures: Bone and Arch. Facial Plast Surg. 2015. 31(4):368-75

Manganello, C. L. S., Silva, A. A. F. \& Pacheco, D. F. S. (2003). Fraturas zigomáticas e orbitozigomáticas. Rev Bras Cir Plást. 18:24-30.

Padmanavam A. \& Mishra S. Patient. (2018). Perspective in the Management of Zygomatic Fractures. Ann Maxillofac Surg; 8(2):239-246 
Research, Society and Development, v. 10, n. 9, e51210918300, 2021

(CC BY 4.0) | ISSN 2525-3409 | DOI: http://dx.doi.org/10.33448/rsd-v10i9.18300

Paulesini Junior, W., Farias, L.P., Aquati, M., Rapoporat, A. \& Leporace, A. A (2008). Fratura de complexo zigomático: relato de caso. Revista de odontologia da universidade cidade de são Paulo 20:301-306.

Peron, M. F., Ferreira, G. M., Camarini, E. T., Iwaki Filho, L., Farah, G. J. \& Pavan, A. J (2009). Epidemiological surveying of the fractures of the zigomatic complex in the Residence Service in Surgery and Bucomaxillofacial Traumatology of UEM in the period of 2005 to 2006. Rev Odontol UNESP; 38(1): 1-5.

Santos Júnior, P. V. (1996). Avaliação clínica comparativa entre as técnicas do gancho de Ginestet e do parafuso de carol girard para o tratamento de fratura simples (afundamento) do osso zigomático. Rev Odontol UNESP; 25(2): 319-325.

Starch-Jensen, T., Linnebjerg, L. B \& Jensen, J. D. (2018). Treatment of zygomatic complex fracture with surgical or nonsurgical intervention: a retrospective study. Open Dent J. 12:377-387. 